\title{
STUDY OF COMPONENT MODE SYNTHESIS METHODS IN A ROTOR-STATOR INTERACTION CASE
}

\author{
A. Batailly, GeM, École Centrale de Nantes, France \\ M. Legrand, Laboratoire de dynamique des structures et vibrations, \\ Université McGill, Québec \\ P. Cartraud, GeM, École Centrale de Nantes, France \\ C. Pierre, Laboratoire de dynamique des structures et vibrations, \\ Université McGill, Québec \\ J.-P. Lombard, Snecma, Site de Villaroche, France
}

Cet article fait partie des actes de la conférence ASME DETC 2007

\begin{abstract}
The study of rotor-stator interactions between blade-tips and outer casings through direct contact in modern turbomachines is very time-consuming if the classical finite element method is used. In order to improve the knowledge over these interaction phenomena, faster methods have to be applied. The construction of reduced-order models using component mode synthesis methods generally allows for dramatic increase in computational efficiency. Two of these methods, namely a fixed interface method and a free interface methods are considered in an original manner to reduce the size of a realistic two-dimensional model. They are then compared in a very specific contact case-study. The equations of motion are solved using an explicit time integration scheme with the Lagrange multiplier method where friction is accounted for. The primary goal of the present study is to investigate the general behavior of such approaches in the presence of contact nonlinearities. It will be shown that in our contact case, a good accuracy can be obtained from a reduced models with very limited number of modes.
\end{abstract}

\section{Introduction}

In modern turbo machines such as aircraft jet engines, structural contacts between the casing and bladed disk are nonlinear interactions that can be responsible for severe damage. Studying such interactions using finite element methods can be very computationally expensive. One way to reduce the size of the problem is to perform a modal analysis and truncate the new basis to a few modes only [1][2]. A different and more accurate modal basis can be constructed by using component mode synthesis methods (CMS). This study will be focused on the use of two CMS methods: the Craig-Bampton 
method [3] which considers fixed interface component modes and the CraigMartinez method [4] which exploits free interface attachment modes. The reduced system of equations of motion are solved using an explicit time integration scheme in conjunction with the Lagrange multiplier that account for contact constraints in the normal as well as the tangential directions. a component mode sensitivity analysis is conducted for each method and respective results are compared to a reference - non reduced - solution.

\section{Modeling}

The 2D finite element models of the bladed disk and the casing are now presented. The model is realistic in such a way that both normal contact and friction forces treatment between the casing and the tip of the blades are allowed. The work of Legrand [1] allows us to validate the reliability of the description.

\subsection{Bladed disk:}

The description of the bladed disk is quite similar to the one used by Legrand [1]. The main difference is the coupling of the 22 blades which is performed through curved beams. The number of curved beams along the radius of the disk is set to one in this study as depicted in Fig.1. Each blade is described with the same number of 6 dof Euler-Bernoulli beams. The global curvature of the blade is obtained by imposing an angle $a_{i}$ between beams $i$ and $i+1$. The angle between two blades is denoted by $\phi$ with $\phi=2 \pi / 22$.

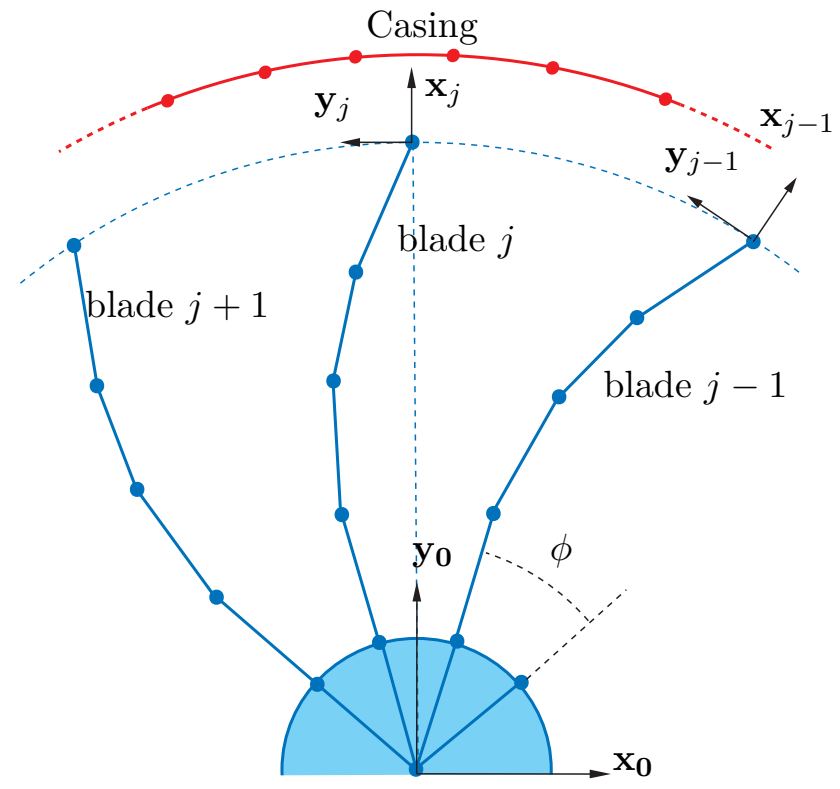

Figure 1 - Two-dimensional model used in the study 


\subsection{Casing:}

The casing is a thin cylinder and is described in this study using two-nodes curved beam finite elements. A polar coordinate system with unit vectors

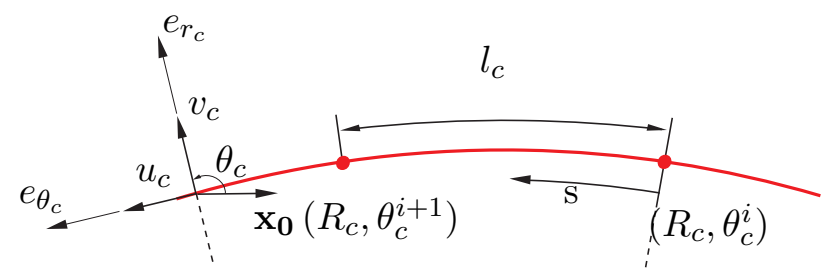

Figure 2 - Detail of a curved beam on the casing between node $i$ and $i+1$

$e_{r_{c}}$ and $e_{\theta_{c}}$ is assigned to the casing. The finite element description involves four dofs per node: $u_{c}, u_{c, s}, v_{c}$ and $v_{c, s}$ as shown on Fig. 2. The initial location of node $i$ is given by the doublet $\left(R_{c}, \theta_{c}^{(i)}\right), R_{c}$ being the radius of the casing and $l_{c}$ the length of the element.

\subsection{Physical analysis of the model:}

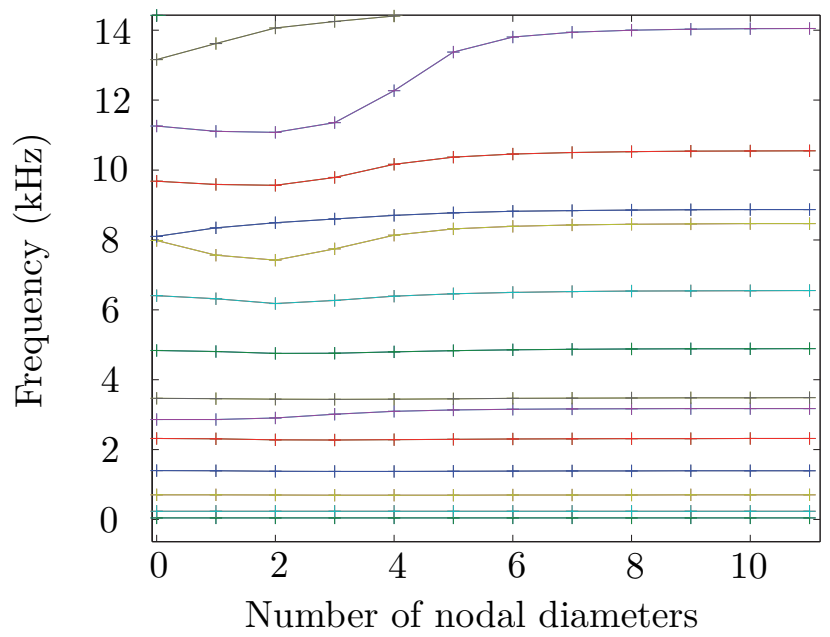

Figure 3 - Distribution of the natural frequencies regarding to the number of nodal diameters

To assess the physical model the repartition of the eigenfrequencies as a function of the number of nodal diameters for the free vibration modes for the blades is shown in Fig. 3. Each curve of this figure is associated with an original bladed disk vibration mode. For instance, the line at $44 \mathrm{~Hz}$ corresponds to the first bending mode of the blades. For higher frequencies, the curves tend to incurve, which results from the apparition of the vibration modes of the disk. One can also check that the lower eigenfrequencies values 
are contained by groups of 22 (the number of blades) close frequencies.

\section{Numerical solving:}

The numerical methods are introduced in this section and described in the general framework of the finite element methods. This part is devoted to the computation of the reference solution. Damping will not be considered in this study in order to keep a good correspondence between the non reduced finite element solution and the reduced solution. The introduction of damping in a reduced model will be done in further work. The general problem to be solved may be written as:

$$
\begin{aligned}
& \mathbf{M} \ddot{\mathbf{u}}+\mathbf{K u}=\mathbf{F} \\
& \mathbf{u}\left(t=t_{0}\right)=\mathbf{u}_{0} \\
& \dot{\mathbf{u}}\left(t=t_{0}\right)=\dot{\mathbf{u}}_{0}
\end{aligned}
$$

The contact conditions, referred to as the Kuhn-Tucker optimality conditions take the form:

$$
\forall \mathbf{x} \in \Gamma_{c}^{m}, \mathbf{t}_{\mathbf{N}} \geq 0, g \geq 0, \mathbf{t}_{\mathbf{N}} g=0
$$

where $\Gamma_{c}^{m}$ is the master surface (bladed disk) and $\mathbf{t}_{\mathbf{N}}$ stands for the contact pressure, assumed positive, acting on the slave surface $\Gamma_{c}^{(s)}$ (casing). This problem is solved using explicit central differences scheme and the forward increment Lagrangian method for managing the contact interactions [5][6]. This procedure is detailed in the study of Legrand [1]. At each time step, a displacement prediction is computed assuming that no contact occurs. Then the gap function is determined between the casing and the bladed disk. If the gap is found to be negative, a correction of the displacement is made, through the contact forces. Friction forces, obtained thanks to the Coulomb's law are also applied.

\section{Modal reduction}

\subsection{General aspect:}

In most industrial applications, the finite element models contain a huge number of degrees of freedom (dofs) leading to cumbersome computation times. One way to reduce these computation times relies in the use of component mode synthesis procedures. Many studies of these CMS methods have been carried out by coupling them with substructuring approaches[7]. Only the modal reduction aspect is considered here: the bladed disk will be reduced considering no substructures, we apply CMS method in the particular case where the whole structure is seen as a substructure. It first requires the separation of the dofs of the structure within two distinct groups: the 


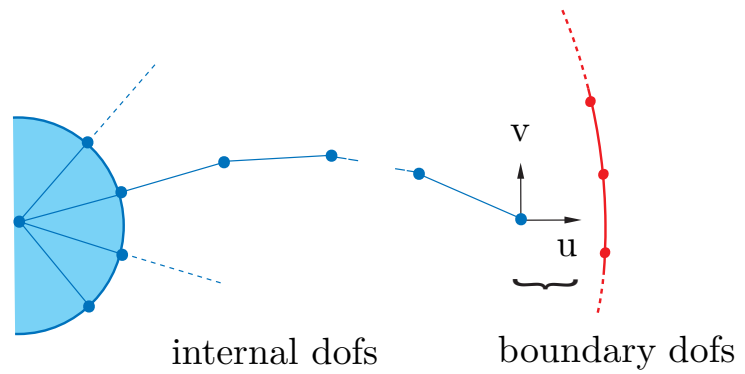

Figure 4 - Decomposition of the blade between boundary and internal dofs

internal dofs and the boundary dofs. The definition of these groups is closely related to the loadings applied on the structures. In our case the boundary is supposed to contain all the dofs that might be supporting any contact force during the simulation. Increasing the set of boundary dofs would not have any impact on the quality of the reduction but would lead to longer computation times (see Fig. 4 for the description of the internal and boundary dofs in our case). In order to maximize the reduction, the only dofs of displacement $u$ and $v$ at the tip of the blades are kept in the model. The other crucial aspect of CMS methods is the choice of the modes included in the reduction basis. Classically, different kinds of modes are considered according to the method used to obtain the transformation matrices. The reduced matrices are then calculated with the transformation matrices and the finite element matrices. In the case of the study of interaction between the casing and the bladed disk as described in section 3, correction forces have to be applied on the tips of the blades (Lagrange multiplier method). Therefore, the corresponding dofs have to be directly accessible in the reduced space in order to avoid permanent backward and forward mapping to the physical space. This requires specific CMS methods such as the Craig-Bampton and Craig-Chang-Martinez ones which are detailed in the following.

\subsection{The Craig-Bampton method :}

The Craig-Bampton method [3] is probably the most popular CMS method from a numerical point of view. The reduction basis of the Craig-Bampton method is composed of fixed interface modes and constraint modes:

- the fixed interface modes are obtained by solving the eigenvalue problem with fixed boundary.

- the constraint modes are as numerous as the number of dofs on the boundary. The constraint mode $i$ is the static response of the structure to a unit displacement of dof $i$ on the boundary while the other ones on the boundary are set to ' 0 '. 
All the constraint modes have to be included in the reduction basis but the number of fixed interface modes kept is variable and its number can be modified to increase the quality of the reduction. A reorganization of Eqn. (1) is necessary, separating the dofs in two groups: the internal dofs $\left(\mathbf{q}_{\mathbf{I}}\right)$ and the external dofs (on the boundary: $\mathbf{q}_{\mathbf{B}}$ ). This yields:

$$
\left[\begin{array}{ll}
\mathbf{M}_{\mathrm{II}} & \mathbf{M}_{\mathrm{BI}} \\
\mathbf{M}_{\mathrm{IB}} & \mathbf{M}_{\mathrm{BB}}
\end{array}\right]\left(\begin{array}{c}
\ddot{\mathrm{q}}_{\mathrm{I}} \\
\ddot{\mathrm{q}}_{\mathrm{B}}
\end{array}\right)+\left[\begin{array}{cc}
\mathbf{K}_{\mathrm{II}} & \mathbf{K}_{\mathrm{BI}} \\
\mathbf{K}_{\mathrm{IB}} & \mathbf{K}_{\mathrm{BB}}
\end{array}\right]\left(\begin{array}{c}
\mathbf{q}_{\mathrm{I}} \\
\mathbf{q}_{\mathrm{B}}
\end{array}\right)=\left(\begin{array}{c}
\mathbf{F}_{\mathrm{I}} \\
\mathbf{F}_{\mathrm{B}}
\end{array}\right)
$$

The reduction matrix of the Craig Bampton method $\Phi_{\mathbf{C B}}$ is:

$$
\begin{gathered}
\Phi_{\mathbf{C B}}=\left[\Phi_{\text {fixed }} \Phi_{\text {constraint }}\right] \\
\left(\begin{array}{c}
\mathbf{q}_{\mathbf{I}} \\
\mathbf{q}_{\mathbf{B}}
\end{array}\right)=\Phi_{\mathbf{C B}}\left(\begin{array}{c}
\mathbf{u}_{\eta} \\
\mathbf{q}_{\mathbf{B}}
\end{array}\right)
\end{gathered}
$$

The projection of the equations of motion in the reduced space consists in the following operations:

$$
\hat{\mathbf{K}}=\Phi_{\mathbf{C B}}^{\mathrm{T}} \mathbf{K} \Phi_{\mathbf{C B}} \text { and } \hat{\mathbf{M}}=\Phi_{\mathbf{C B}}^{\mathrm{T}} \mathbf{M} \Phi_{\mathbf{C B}}
$$

Consequently, the dynamic equation in the new space becomes:

$$
\hat{\mathbf{M}}\left(\begin{array}{c}
\ddot{\mathbf{u}}_{\eta} \\
\ddot{\mathbf{q}}_{\mathbf{B}}
\end{array}\right)+\hat{\mathbf{K}}\left(\begin{array}{c}
\mathbf{u}_{\eta} \\
\mathbf{q}_{\mathbf{B}}
\end{array}\right)=\left(\begin{array}{c}
\mathbf{F}_{\eta} \\
\mathbf{F}_{\mathbf{B}}
\end{array}\right)
$$
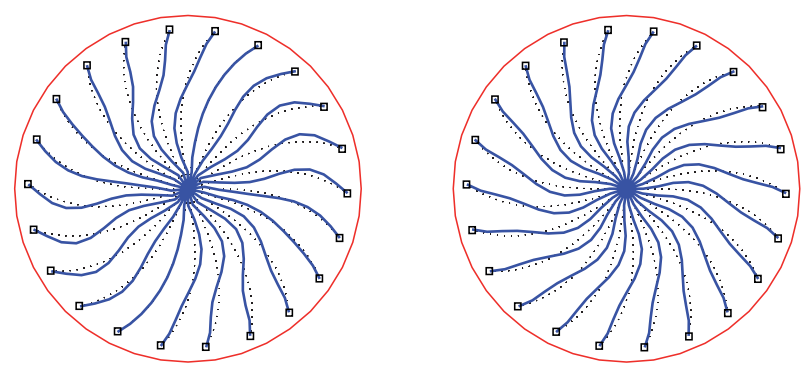

Figure 5 - Examples of fixed interface modes part of the bladed disk. The squares located at the blade tips mean that the dofs of these nodes are fixed.

\subsection{The Craig-Chang-Martinez method:}

This method is actually an enriched Craig-Martinez method [4][8]. The reduction basis is composed of free vibration modes (which number is variable) and attachment modes:

- free vibration modes are obtained by solving the initial eigenvalue problem. 

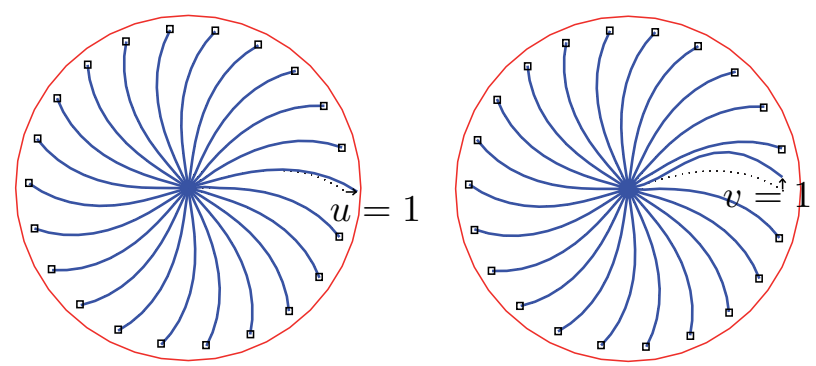

Figure 6 - Examples of constraint modes (for the dofs $u$ and $v$ on the blade 2) composing the reduction basis of the bladed disk.

- attachment modes: their number is the same as the number of dofs in the boundary. The attachment mode $j$ is the static response of the structure to a unit external loading on dof $j$.

From a theoretical point of view, there is no restriction in the choice of these modes. From Eqn. (1), the projection of the displacements $\mathbf{u}$ onto the modal coordinates leads to:

$$
\mathbf{u}=\Phi_{1} \mathbf{u}_{\mathbf{1}}+\Phi_{2} \mathbf{u}_{\mathbf{2}}
$$

where $\Phi_{1}$ represents the free vibration modes kept in the reduction basis and $\Phi_{2}$ the modes that will not be retained for the reduction (high frequency modes).

$$
\mathbf{M}\left(\Phi_{1} \ddot{\mathbf{u}}_{\mathbf{1}}+\Phi_{2} \ddot{\mathbf{u}}_{\mathbf{2}}\right)+\mathbf{K}\left(\Phi_{1} \mathbf{u}_{\mathbf{1}}+\Phi_{2} \mathbf{u}_{\mathbf{2}}\right)=\mathbf{F}
$$

Eqn. (9) is then multiplied by $\Phi_{1}^{\mathrm{T}}$ then by $\Phi_{2}^{\mathrm{T}}$. The $\mathbf{K}$ and $\mathbf{M}$-orthogonality of modes $\Phi_{i}$ yields:

$$
\begin{aligned}
\left(\boldsymbol{\Omega}_{1}-\omega^{2} \mathbf{I}\right) \mathbf{u}_{\mathbf{1}} & =\Phi_{1}{ }^{\mathrm{T}} \mathbf{F} \\
\left(\Omega_{2}-\omega^{2} \mathbf{I}\right) \mathbf{u}_{\mathbf{2}} & =\Phi_{2}{ }^{\mathrm{T}} \mathbf{F}
\end{aligned}
$$

where $\Omega_{1}$ and $\Omega_{2}$ are the diagonal matrices containing the eigenvalues associated with matrices $\Phi_{1}$ and $\Phi_{2}$. The 'pseudo-static' approximation is assumed considering that the lowest frequency of $\Phi_{2}$ is definitely higher than the maximum frequency considered in the study of the structure:

$$
\forall \omega_{2} \in \operatorname{diag}\left(\Omega_{2}\right), \omega \ll \omega_{2} \Rightarrow \Omega_{2} \mathbf{u}_{2}=\Phi_{2}^{\mathrm{T}} \mathbf{F}
$$

Using the decomposition of $\mathbf{u}$ in a static formulation, we obtain:

$$
\Phi_{2} \mathbf{u}_{\mathbf{2}}=\mathbf{K}^{-\mathbf{1}} \mathbf{F}-\Phi_{1} \mathbf{u}_{\mathbf{1}}
$$

Consequently Eqn. (10) become $\Omega_{1} \mathbf{u}_{\mathbf{1}}=\Phi_{1}^{\mathrm{T}} \mathbf{F}$ or, equivalently:

$$
\mathbf{u}_{\mathbf{1}}=\Omega_{1}^{-1} \Phi_{1}^{\mathrm{T}} \mathbf{F}
$$

Then, plugging Eqn. (13) into Eqn. (12) yields:

$$
\Phi_{2} \mathbf{u}_{2}=\left(\mathbf{K}^{-1}-\Phi_{1} \Omega_{1}^{-1} \Phi_{1}^{\mathrm{T}}\right) \mathbf{F}
$$


The term $\mathbf{K}^{-1}-\Phi_{1} \Omega_{1}^{-1} \Phi_{1}^{\mathrm{T}}$ is called residual static flexibility and is denoted by $\mathbf{R}$. We finally get:

$$
\mathbf{u}=\Phi_{1} \mathbf{u}_{\mathbf{1}}+\mathbf{R F}
$$

Eqn. (15) is the starting point of the Craig-Martinez method. The goal of this method is to obtain the boundary dofs in the parameters of the reduced system. This is achieved by modifying the expression of the external forces $\mathbf{F}$ in the previous equations. $\mathbf{u}$ will now be reorganized such as $\mathbf{u}=\left(\mathbf{q}_{\mathbf{B}}, \mathbf{q}_{\mathbf{I}}\right)^{\mathrm{T}}$. Then Eqn. (15) takes the from:

$$
\left(\begin{array}{c}
\mathbf{q}_{\mathbf{B}} \\
\mathbf{q}_{\mathbf{I}}
\end{array}\right)=\left[\begin{array}{c}
\Phi_{1 B} \\
\Phi_{1 I}
\end{array}\right] \mathbf{u}_{\mathbf{1}}+\left[\begin{array}{c}
\mathbf{R}_{\mathbf{B}} \\
\mathbf{R}_{\mathbf{I}}
\end{array}\right] \mathbf{F}
$$

The notations will be simplified here, $\mathbf{R}_{\mathbf{B}}$ and $\mathbf{R}_{\mathbf{I}}$ are blocks of the square matrix $\mathbf{R}$. However, since $\mathbf{F}$ will only have coordinates on the external dofs (the external dofs are chosen to be the only one being loaded) we can write:

$$
\left[\begin{array}{c}
\mathbf{R}_{\mathbf{B}} \\
\mathbf{R}_{\mathbf{I}}
\end{array}\right] \mathbf{F} \Leftrightarrow\left[\begin{array}{cc}
\mathbf{R}_{\mathbf{B} 1} & \mathbf{R}_{\mathrm{B} 2} \\
\mathbf{R}_{\mathbf{I} 1} & \mathbf{R}_{\mathbf{I} 2}
\end{array}\right]\left(\begin{array}{c}
\mathbf{F}_{\mathbf{1}} \\
\mathbf{0}
\end{array}\right)
$$

and from now on we denote: $\mathbf{R}_{\mathbf{B}}=\mathbf{R}_{\mathbf{B} \mathbf{1}}$ and $\mathbf{R}_{\mathbf{I}}=\mathbf{R}_{\mathbf{I} \mathbf{1}}$. Eqn. (16) becomes then:

$$
\left(\begin{array}{l}
\mathbf{q}_{\mathbf{B}} \\
\mathbf{q}_{\mathbf{I}}
\end{array}\right)=\left[\begin{array}{ll}
\Phi_{1 B} & \mathbf{R}_{\mathbf{B}} \\
\Phi_{1 I} & \mathbf{R}_{\mathbf{I}}
\end{array}\right]\left(\begin{array}{c}
\mathbf{u}_{\mathbf{1}} \\
\mathbf{F}
\end{array}\right)
$$

The first block of Eqn. (18) is:

$$
\mathbf{F}=\mathbf{R}_{\mathbf{B}}{ }^{-1}\left(\mathbf{q}_{\mathbf{B}}-\Phi_{1 B} \mathbf{u}_{\mathbf{1}}\right)
$$

This equation allows us to introduce the external dofs in the reduced system:

$$
\left(\begin{array}{l}
\mathbf{q}_{\mathbf{B}} \\
\mathbf{q}_{\mathbf{I}}
\end{array}\right)=\underbrace{\left[\begin{array}{cc}
\mathbf{I}_{\mathbf{B f}} & \mathbf{0} \\
\mathbf{R}_{\mathbf{I}} \mathbf{R}_{\mathbf{B}}^{-1} & \Phi_{1 i}-\mathbf{R}_{\mathbf{I}} \mathbf{R}_{\mathbf{B}}{ }^{-1} \Phi_{1 f}
\end{array}\right]}_{\mathbf{P}_{\mathbf{C M}}}\left(\begin{array}{l}
\mathbf{q}_{\mathbf{B}} \\
\mathbf{u}_{\mathbf{1}}
\end{array}\right)
$$

This way, $\mathbf{P}_{\mathbf{C M}}$ is defined as the reduction matrix for the Craig-Martinezmethod. The Craig-Chang-Martinez method is very similar to the CraigMartinez method, the only difference is in the definition of the reduction basis $\Phi_{1}$ that is enriched by the attachement modes. The following list details the coordinates of the force vectors applied in Fig. 7:

- $\mathbf{F}_{\mathbf{y}}=0 \cdot \mathbf{x}+1 \cdot \mathbf{y}+0 \cdot \mathbf{z}$

- $\mathbf{F}_{\mathbf{x}}=1 \cdot \mathbf{x}+0 \cdot \mathbf{y}+0 \cdot \mathbf{z}$ 


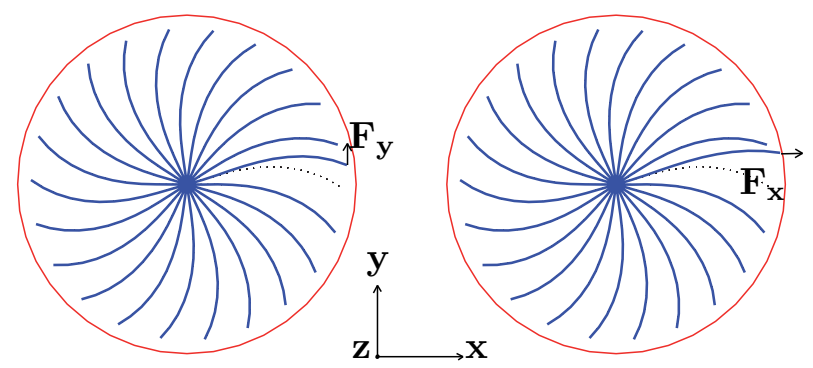

Figure 7 - Examples of attachment modes (efforts applied on the second blade) of the bladed disk.
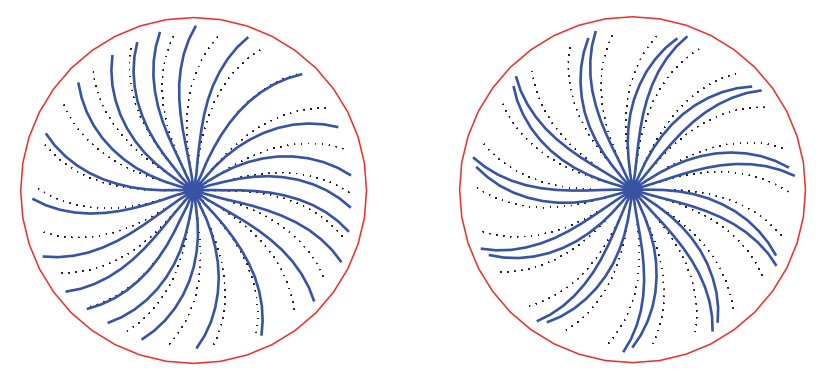

Figure 8 - Examples of free vibration modes ( 3 and 11 diameters) of the bladed disk.

\section{Influence of the reduction modes}

\subsection{Parameters of the study:}

The disk has 22 blades described by 10 beams each (one for the disk part and 9 for the blade itself), table 1 gives the geometrical description of the structure. The two-dimensional model contains 748 dofs: 44 external dofs (displacements $u$ and $v$ on each tips of the blades) and 704 internal dofs. The casing is described by 40 curved beams and cannot be reduced since contact efforts can be exert anywhere on the structure. Initially, the clearance between the tip of the blades and the casing is $1 \mathrm{~mm}$, the bladed disk is rotating at the speed $\omega=310 \mathrm{rad} \cdot \mathrm{s}^{-1}$. At time $t=0$ a two-nodal diameter external forcing $F_{2 d}$ is applied on the casing over $2 \cdot 10^{-4} \mathrm{~s}$. The simulation time is $20 \mathrm{~ms}$, over which one we collect the data every 50 time steps. The time step $\Delta t$ of the explicit central differences scheme is set to $5 \cdot 10^{-7} \mathrm{~s}$ with respect to the following condition which is classical in linear analysis:

$$
\Delta t<\frac{2}{\omega_{\max }}=8.49 \cdot 10^{-7} \mathrm{~s}
$$

The goal of this study is to evaluate the influence of every component mode used in CMS methods. We will also consider the computation time of each method. 


\begin{tabular}{|r|r|}
\hline Number of blades & 22 \\
\hline Radius of the bladed disk & $500 \mathrm{~mm}$ \\
\hline Radius $R_{c}$ of the casing & $501 \mathrm{~mm}$ \\
\hline Angular velocity & $310 \mathrm{rad} \cdot \mathrm{s}^{-1}$ \\
\hline
\end{tabular}

Table 1 - Parameters of the contact study

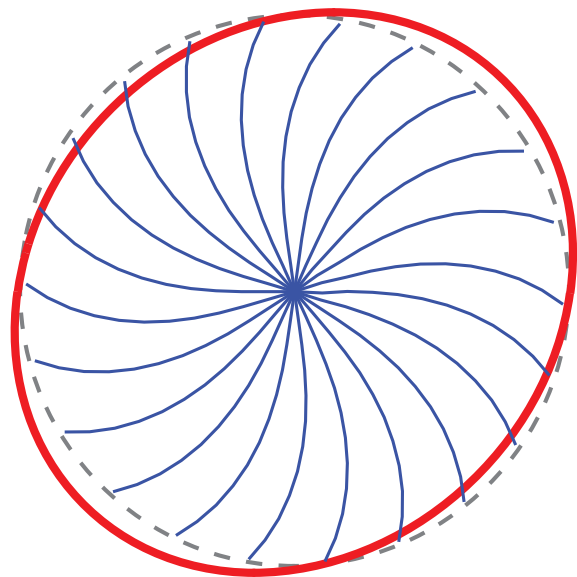

Static deformation of the casing under $F_{2 d}$

- - - Initial shape of the casing

Figure 9 - Deformation of the casing under the $F_{2 d}$ load.

\subsection{Craig-Bampton convergence}

The constraint modes considered here are the ones linked with the displacement dofs $(u$ and $v)$ at the tip of each blades. The parameter of the study is the number $\eta$ of fixed-interface modes in the reduction basis. In order to outline the contact phenomena, the radial gap of the blade tip 1 is computed using the Craig-Bampton method. This gap is denoted by $u_{c b}(\eta, t)$ and depends on time and $\eta$. This gap is compared to the reference one obtained by classical finite element analysis $u_{e f}(t)$.

\subsection{Craig-Chang-Martinez convergence}

For the Craig-Chang-Martinez method, attachment modes are considered relatively to the dofs used for the constraint modes in the Craig-Bampton method meaning the number of attachment modes (44) is the same as the number of constraint modes in the previous section. The choice of the attachment is very sensitive in the Craig-Chang-Martinez method. This method can be instable depending on the choice of these modes [9]: for instance, in our specific case, the use of the attachment modes linked to rotation dofs $(\theta)$ leads to ill-conditioned matrices. The parameter of the 


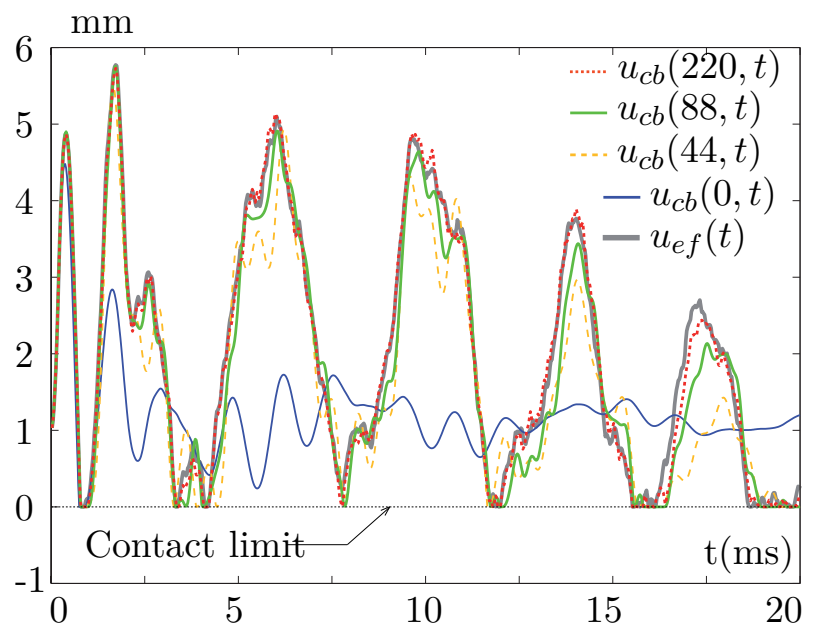

Figure 10 - Distance between blade tip 1 and the casing for the CraigBampton method [0;20ms].

study is the number of free vibration modes $\psi$ kept in the reduction basis. In the same way as for the Craig-Bampton study, we will represent the radial gap between the blade tip 1 along the axis $\mathbf{x}_{\mathbf{1}}$ (see Fig. 1). This gap is denoted $u_{c c m}(\psi, t)$ and compared to $u_{e f}(t)$.

\subsection{Comparison of the two CMS methods}

The very principle of using component mode synthesis is reducing the dimension of the space while keeping accurate results. With the minimum number of modes in each method (44 constraint modes for Craig-Bampton and 44 attachment modes for Craig-Chang-Martinez), we can clearly differentiate the results obtained by the two methods. The curve of $u_{c b}(0, t)$ in Figs. 10 and 11 shows that the constraint modes cannot be used alone to describe accurately the displacement of the blade. On the contrary the curve of $u_{c c m}(0, t)$ on Figs. 12 and 13 shows that the attachment modes give a good approximation of the finite element solution. For higher number of modes in each reduction basis $(\psi>0$ and $\eta>0)$ both methods give similar results converging through the finite element solution. In terms of calculation time, the decrease obtained with the use of CMS method is represented in Fig. 14. On the computer used for the calculations, the reference time for finite element calculation was $1031 \mathrm{~s}$. The size of the model is still relatively small. Better results are expected for large 3D models.

\section{Conclusion}

This study shows that linear component mode synthesis methods can be used successfully in non-linear cases such as rotor-stator interaction. The first results obtained without damping allow us to conclude that the attach- 


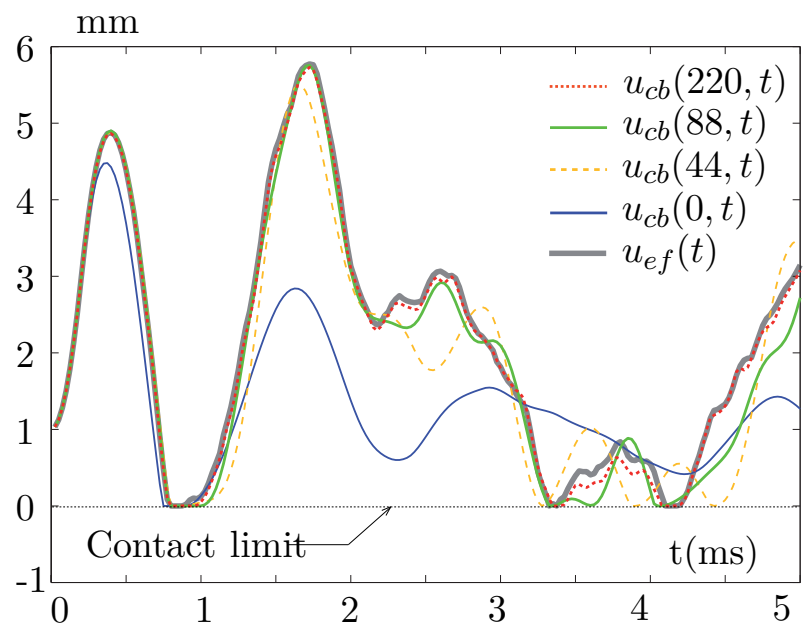

Figure 11 - Distance between blade tip 1 and the casing for the CraigBampton method $[0 ; 5 \mathrm{~ms}]$.

ment modes used in the Craig-Chang-Martinez method give a very good approximation of the displacements at the top of the blades. In terms of computation time, keeping only these attachment modes decreases computation time by $84.4 \%$ (see Fig.14) from the classical finite element solution to the Craig-Chang-Martinez solution (the decreasing being $81.5 \%$ when 88 free vibration modes are added). For a larger number of modes, the solution given by the Craig-Bampton method and the Craig-Chang-Martinez method are very similar even though the convergence speed of the CraigBampton method seems higher. The gain in computation time will increase when more realistic 3D models will be considered for industrial applications. Further work has to be done for optimizing the selection of the modes in the reduction basis for each CMS method and work is in progress to introduce damping.

\section{Acknowledgment}

Thanks go to Snecma for its technical and financial support. This work takes place in the framework of the MAIA mechanical research and technology program sponsored by CNRS, ONERA and SAFRAN Group.

\section{References}

[1] M. Legrand, C. Pierre, P. Cartraud. "Numerical modeling of an aircraft engine structural rotor-stator modal interaction". Submitted.

[2] M. Legrand, 2005. "Modeles de prediction de l'interaction rotor/stator dans un moteur d'avion". PhD Thesis, Ecole Centrale de Nantes, Nantes, FRANCE. 


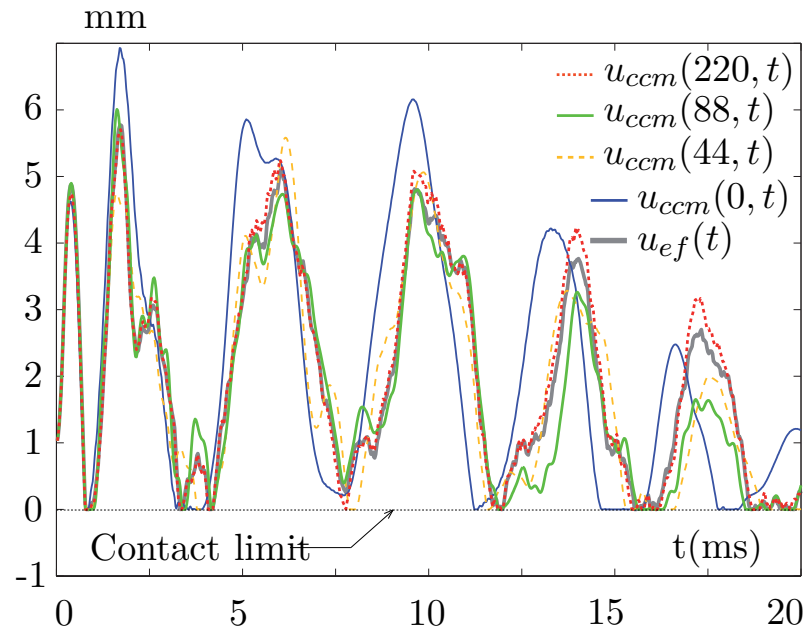

Figure 12 - Distance between blade tip 1 and the casing for the CraigChang-Martinez method $[0 ; 20 \mathrm{~ms}]$.

[3] Roy R. Craig Jr. and Mervyn C. C. Bampton, 1968. "Coupling of Substructures for Dynamic Analyses". AIAA Journal, 6(7), July, pp. 13141319 .

[4] D. M. Tran, 2000. "Component mode synthesis methods using interface modes. Application to structures with cyclic symmetry". Computers \& Structures , 79, pp. 209-222.

[5] J. C. Simo and T. A. Laursen, 1992. "An augmented lagrangian treatment of contact problems involving friction". Computers \& Structures, 42(1), pp. 97-116.

[6] N. Carpenter, R. Taylor and M. Katona, 1991. "Lagrange constraints for transcient finite element surface contact". International Journal for Numerical Methods in Engineering, 32, pp. 103-128.

[7] D. J. Rixen, 2004. "A dual Craig-Bampton method for dynamic substructuring". Journal of Computational and applied mathematics, 168, pp. 383-391.

[8] M. A. Tournour, N. Atalla, O. Chiello, F. Sgard, 2001. "Validation, performance, convergence and application of free interface component mode synthesis". Computers \& Structures, 79, pp. 1861-1876.

[9] R. Bladh, C. Pierre, and M. P. Castanier, 2003. "Numerical Instability of Classical Free-Interface Component Mode Synthesis Techniques". AIAA Journal, 41(8), pp. 1621-1624. 


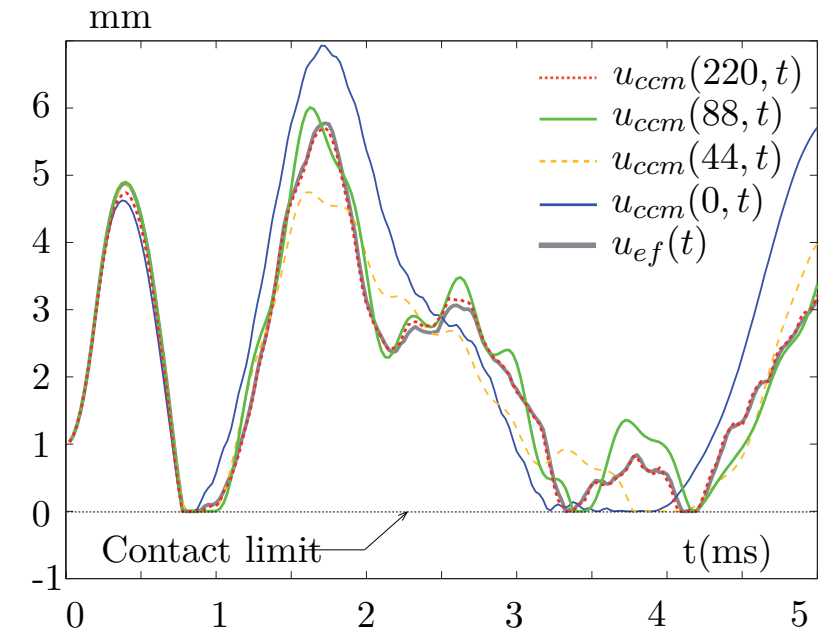

Figure 13 - Distance between blade tip 1 and the casing for the CraigChang-Martinez method $[0 ; 5 \mathrm{~ms}]$.

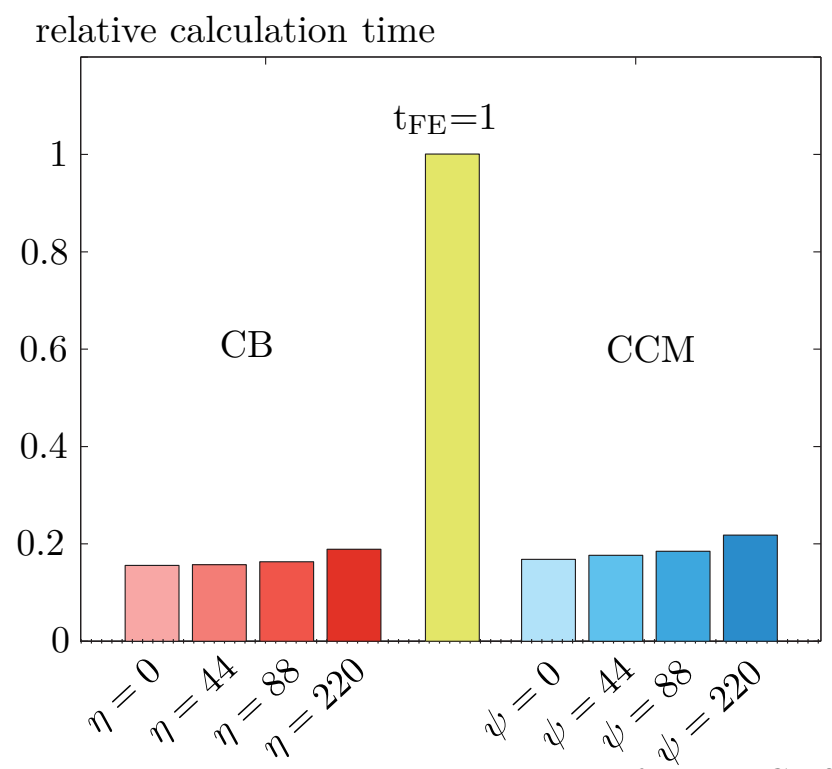

Figure 14 - Relative calculation times obtained for each CMS method. 